\title{
LINEAR SYSTEMS OF RATIONAL CURVES ON RATIONAL SURFACES
}

\author{
DANIEL DAIGLE, ALEJANDRO MELLE-HERNÁNDEZ \\ Dedicated to the memory of Vladimir Igorevich Arnold
}

Let $S$ be a projective nonsingular rational surface, over an algebraically closed field of characteristic zero. We say that a linear system $\mathbb{L}$ on $S$ is rational if $\operatorname{dim} \mathbb{L} \geq 1$ and the general member of $\mathbb{L}$ is an irreducible rational curve.

Let $C \subset S$ be an irreducible curve.

Let $\Omega_{C}$ be the set of rational linear systems $\mathbb{L}$ on $S$ satisfying $C \in \mathbb{L}$. Consider the minimal 11 resolution of singularities $\pi: \widetilde{S} \rightarrow S$ of $C$, let $\widetilde{C}$ be the strict transform of $C$ on $\widetilde{S}$, and let $\tilde{\nu}(C)$ denote the self-intersection number of $\widetilde{C}$ in $\widetilde{S}$. Then Theorem 2.8 implies:

(1) $\Omega_{C} \neq \varnothing$ if and only if $C$ is rational and $\tilde{\nu}(C) \geq 0$.

Let $\mathbb{L}_{C}$ be the linear system on $S$ which is the image of $|\widetilde{C}|$ by $\pi_{*}: \operatorname{Div}(\widetilde{S}) \rightarrow \operatorname{Div}(S)$ (so $C \in \mathbb{L}_{C}$ ). Assuming that $\Omega_{C} \neq \varnothing$, we show (Thm 2.8):

(2) For any linear system $\mathbb{L}$ on $S, \mathbb{L} \in \Omega_{C} \Longleftrightarrow \operatorname{dim} \mathbb{L} \geq 1$ and $C \in \mathbb{L} \subseteq \mathbb{L}_{C}$.

This gives a complete description of $\Omega_{C}$, and we note in particular that $\Omega_{C}$ has a greatest element (namely, $\mathbb{L}_{C}$ ). Continuing to assume that $\Omega_{C} \neq \varnothing$, Theorem 2.8 also shows that $\operatorname{dim} \mathbb{L}_{C}=\tilde{\nu}(C)+1$ and that the minimal resolution of singularities of $C$ coincides with the minimal resolution of the base points of $\mathbb{L}_{C}$.

The present paper may be viewed as a preamble to the forthcoming [2], in which we study linear systems associated to unicuspidal rational curves $C \subset \mathbb{P}^{2}$. We remind the reader that all currently known curves of that type satisfy $\tilde{\nu}(C) \geq 0$, hence $\Omega_{C} \neq \varnothing$. It is shown in 2] that if $C \subset \mathbb{P}^{2}$ is a unicuspidal rational curve with singular point $P$ then: (1) there exists a unique pencil $\Lambda_{C}$ on $\mathbb{P}^{2}$ satisfying $C \in \Lambda_{C}$ and $\operatorname{Bs}\left(\Lambda_{C}\right)=\{P\}$; (2) $\Lambda_{C}$ is a rational pencil if and only if $\tilde{\nu}(C) \geq 0$; (3) if $\tilde{\nu}(C) \geq 0$, then $\Lambda_{C}$ has a dicritical of degree 1 . From these facts, the paper then derives consequences for the classification of unicuspidal rational curves $C \subset \mathbb{P}^{2}$ satisfying $\tilde{\nu}(C) \geq 0$.

We would like to express our thanks to Professor Dolgachev, and also to the referee, for their useful comments.

2010 Mathematics Subject Classification. Primary: 14C20. Secondary: 14J26.

Date: January 18, 2019.

Research of the first author supported by a grant from NSERC Canada.

Research of the second author supported by the grants MTM2010-21740-C02-01 and Grupo Singular CCG07-UCM/ESP-2695-921020.

${ }^{1}$ This is the "short" resolution, not the "embedded" resolution. See 1.4 for details. 
Conventions. All algebraic varieties are over an algebraically closed field $\mathbf{k}$ of characteristic zero. Varieties are irreducible and reduced, so in particular all curves are irreducible and reduced. A divisor $D$ of a surface is reduced if $D=\sum_{i=1}^{n} C_{i}$ where $C_{1}, \ldots, C_{n}$ are distinct curves $(n \geq 0)$.

\section{Clusters on a surface}

We fix a projective nonsingular surface $S$ throughout this section. We consider the set $S^{*}$ of points which are either points of $S$ or points infinitely near points of $S$. The set $S^{*}$ comes equipped with a partial order $\leq$, called the natural order, such that for $P, Q \in S^{*}$ we have $P<Q$ if and only if $Q$ is infinitely near $P$. The minimal elements of $\left(S^{*}, \leq\right)$ are called the proper points of $S$, and are indeed in bijective correspondence with the closed points of $S$. Note that the poset $\left(S^{*}, \leq\right)$ is a classical object (for instance it is called a "bubble space" in [4] but has the order relation reversed).

A cluster on $S$ is a (possibly empty) finite subset $K \subset S^{*}$ such that, given any $P, Q \in S^{*}$, if $P \leq Q$ and $Q \in K$ then $P \in K$. If $K$ is a cluster on $S$ then a subcluster of $K$ is any subset of $K$ which is itself a cluster on $S$. Note that if $K$ is a cluster on $S$ then each minimal element of $K$ is a proper point of $S$.

The aim of this section is to fix the notations and terminologies for clusters and to recall certain facts in that theory - there are no new results here. Our main reference is the first chapter of [1], and our notations and definitions are in general compatible with that text.

1.1. Let $K$ be a cluster on $S$.

(a) The blowing-up of $S$ along $K$ is denoted $\pi_{K}: S_{K} \rightarrow S$. Observe that if $K^{\prime}$ is a subcluster of $K$ then $K \backslash K^{\prime}$ is a cluster on $S_{K^{\prime}}$ and $\pi_{K}$ factors as

$$
S_{K} \stackrel{\pi_{K \backslash K^{\prime}}}{\longrightarrow} S_{K^{\prime}} \stackrel{\pi_{K^{\prime}}}{\longrightarrow} S .
$$

(b) Given a divisor $D \in \operatorname{Div}(S)$, let $\widetilde{D}^{K} \in \operatorname{Div}\left(S_{K}\right)$ and $\bar{D}^{K} \in \operatorname{Div}\left(S_{K}\right)$ denote, respectively, the strict transform and total transform of $D$ on $S_{K}$.

(c) If $\mathbb{L}$ is a linear system on $S$ without fixed components and such that $\operatorname{dim} \mathbb{L} \geq 1$, let $\widetilde{\mathbb{L}}^{K}$ denote the strict transform of $\mathbb{L}$ on $S_{K}$.

(d) Given $P \in K$, one can define the corresponding exceptional curve $E_{P}$ as follows. Consider the subcluster $K^{\prime}=\{x \in K \mid x \leq P\}$ of $K$ and factor $\pi_{K}$ as in (1). Then $E_{P} \subset S_{K^{\prime}}$ is the unique irreducible component of the exceptional locus of $\pi_{K^{\prime}}$ with self-intersection $(-1)$. The strict transform (resp. total transform) of $E_{P}$ on $S_{K}$ is denoted $\widetilde{E}_{P}^{K} \subset S_{K}\left(\operatorname{resp} . \bar{E}_{P}^{K} \in \operatorname{Div}\left(S_{K}\right)\right)$.

1.2. (a) Given $P \in S^{*}$, consider the blowing-up $\pi_{K^{P}}: S_{K^{P}} \rightarrow S$ of $S$ along the cluster $K^{P}=\left\{x \in S^{*} \mid x<P\right\}$, and note that $P$ is a proper point of $S_{K^{P}}$.

(b) Given $P \in S^{*}$ and a curve $C \subset S$, let $e_{P}(C) \in \mathbb{N}$ denote the multiplicity of $C$ at $P$ (by definition, this is the multiplicity of the curve $\widetilde{C}^{K^{P}} \subset S_{K^{P}}$ at the proper point $P$ of $S_{K^{P}}$ ). Extending linearly, let $e_{P}(D) \in \mathbb{Z}$ denote the multiplicity of a divisor $D \in \operatorname{Div}(S)$ at $P$. 
(c) Given $P \in S^{*}$ and a linear system $\mathbb{L}$ on $S$ without fixed components and such that $\operatorname{dim} \mathbb{L} \geq 1$, let $e_{P}(\mathbb{L}) \in \mathbb{N}$ denote the multiplicity of $\mathbb{L}$ at $P$ (by definition, $\left.e_{P}(\mathbb{L})=\min \left\{e_{P}(D) \mid D \in \widetilde{\mathbb{L}}^{K^{P}}\right\}\right)$. Note that the general member $D$ of $\widetilde{\mathbb{L}}^{K^{P}}$ satisfies $e_{P}(D)=e_{P}(\mathbb{L})$.

1.3. A weighted cluster on $S$ is a pair $(K, m)$ where $K$ is a cluster on $S$ and $m: K \rightarrow \mathbb{Z}$ is any set map. If $K^{\prime}$ is a subcluster of $K$ and $m^{\prime}: K^{\prime} \rightarrow \mathbb{Z}$ is the restriction of $m$, we call $\left(K^{\prime}, m^{\prime}\right)$ a weighted subcluster of $(K, m)$.

1.4. Consider an effective divisor $D \in \operatorname{Div}(S)$.

(a) Define the set $K^{D}=\left\{P \in S^{*} \mid e_{P}(D)>1\right\}$ and note that this is a finite set if and only if $D$ is reduced.

(b) Assume that $D$ is reduced. Then $K^{D}$ is a cluster on $S$, called the cluster of singular points of $D$. If $K^{D}=\varnothing$, we say that $D$ is nonsingular. The blowing-up $\pi_{K^{D}}: S_{K^{D}} \rightarrow S$ of $S$ along $K^{D}$ is called the minimal resolution of singularities of $D$. For an arbitrary cluster $K$ on $S$,

$$
\widetilde{D}^{K} \text { is nonsingular } \Longleftrightarrow K^{D} \subseteq K .
$$

(c) Continue to assume that $D$ is reduced. If $e(D): K^{D} \rightarrow \mathbb{Z}$ denotes the map $P \mapsto e_{P}(D)$ then we call $\mathcal{K}^{D}=\left(K^{D}, e(D)\right)$ the weighted cluster of singular points of $D$.

1.5. Consider a linear system $\mathbb{L}$ on $S$ such that $\operatorname{dim} \mathbb{L} \geq 1$ and without fixed components.

(a) The set $K_{\mathbb{L}}=\left\{P \in S^{*} \mid e_{P}(\mathbb{L})>0\right\}$ is a cluster on $S$, called the cluster of base points of $\mathbb{L}$. The blowing-up $\pi_{K_{\mathbb{L}}}: S_{K_{\mathbb{L}}} \rightarrow S$ of $S$ along $K_{\mathbb{L}}$ is called the minimal resolution of the base points of $\mathbb{L}$. For an arbitrary cluster $K$ on $S$,

$$
\widetilde{\mathbb{L}}^{K} \text { is base-point-free } \Longleftrightarrow K_{\mathbb{L}} \subseteq K .
$$

Let us also observe the following property of $K_{\mathbb{L}}$ :

For each $P \in K_{\mathbb{L}}$, if $\left(\widetilde{E}_{P}^{K_{\mathbb{L}}}\right)^{2}=-1$ in $S_{K_{\mathbb{L}}}$ then $\widetilde{E}_{P}^{K_{\mathbb{L}}}$ is a horizontal curve (i.e., is not included in the support of an element of $\widetilde{\mathbb{L}}^{K_{\mathrm{L}}}$ ).

(b) If $e(\mathbb{L}): K_{\mathbb{L}} \rightarrow \mathbb{Z}$ denotes the map $P \mapsto e_{P}(\mathbb{L})$ then we call $\mathcal{K}_{\mathbb{L}}=\left(K_{\mathbb{L}}, e(\mathbb{L})\right)$ the weighted cluster of base points of $\mathbb{L}$.

(c) We write $\operatorname{Bs}(\mathbb{L})=\left\{Q \in S \mid e_{Q}(\mathbb{L})>0\right\}$ for the base locus of $\mathbb{L}$. Note that this is a set of proper points of $S$, and is the set of minimal elements of $K_{\mathbb{L}}$.

1.6. Let $\mathcal{K}=(K, m)$ be a weighted cluster on $S$ and $D$ a divisor on $S$. Let us use the notation $m=\left(m_{P}\right)_{P \in K}$ for the map $m$ and let $\pi_{K}: S_{K} \rightarrow S$ be the blowing-up of $S$ along $K$.

(a) The virtual transform of $D$ with respect to $\mathcal{K}$ is the $\operatorname{divisor} \check{D}^{\mathcal{K}} \in \operatorname{Div}\left(S_{K}\right)$ defined by:

$$
\check{D}^{\mathcal{K}}=\bar{D}^{K}-\sum_{P \in K} m_{P} \bar{E}_{P}^{K}
$$


(b) We say that $D$ goes through $\mathcal{K}$ if $\check{D}^{\mathcal{K}}$ is an effective divisor. Note that if $D$ goes through $\mathcal{K}$ then $D$ is effective.

(c) We say that $D$ goes through $\mathcal{K}$ effectively if the following equivalent conditions are satisfied:

- $D$ is effective and $e_{P}(D)=m_{P}$ for all $P \in K$

- $D$ goes through $\mathcal{K}$ and $e_{P}(D)=m_{P}$ for all $P \in K$

- $D$ is effective and $\check{D}^{\mathcal{K}}=\widetilde{D}^{K}$.

We leave it to the reader to verify assertions 1.71.11, below. To prove 1.11(b), one uses characteristic zero Bertini Theorem.

1.7. Let $\mathcal{K}$ be a weighted cluster on $S$ and $D \in \operatorname{Div}(S)$. If $D$ goes through $\mathcal{K}$, then $D$ goes through every weighted subcluster of $\mathcal{K}$.

1.8. Let $\mathcal{K}=(K, m)$ be a weighted cluster on $S$ and $D \in \operatorname{Div}(S)$. Suppose that $D$ goes through $\mathcal{K}$, and that $e_{P}(D) \leq m_{P}$ for all $P \in K$. Then $e_{P}(D)=m_{P}$ for all $P \in K$.

1.9. Let $\mathbb{L}$ be a linear system on $S$ without fixed component and such that $\operatorname{dim} \mathbb{L} \geq 1$. For any $D \in \mathbb{L}$ and any cluster $K$ on $S$, the following are equivalent:

(a) $\widetilde{D}^{K} \in \widetilde{\mathbb{L}}^{K}$

(b) $e_{P}(D)=e_{P}(\mathbb{L})$ for all $P \in K$

(c) $e_{P}(D) \leq e_{P}(\mathbb{L})$ for all $P \in K$

(d) D goes through the weighted cluster $(K, e(\mathbb{L}))$ effectively, where $e(\mathbb{L}): K \rightarrow \mathbb{Z}$ denotes the set map $P \mapsto e_{P}(\mathbb{L})$.

1.10. Notation. If $\mathcal{K}=(K, m)$ is a weighted cluster, let $\mathcal{K}^{(>1)}=\left(K^{\prime}, m^{\prime}\right)$ be the pair defined by setting $K^{\prime}=\{P \in K \mid m(P)>1\}$ and by letting $m^{\prime}: K^{\prime} \rightarrow \mathbb{Z}$ be the restriction of $m: K \rightarrow \mathbb{Z}$ to $K^{\prime}$.

1.11. Let $\mathbb{L}$ be a linear system on $S$ without fixed component and such that $\operatorname{dim} \mathbb{L} \geq 1$.

(a) For any $D \in \mathbb{L}$, the following are equivalent:

(i) $\mathcal{K}^{D}=\mathcal{K}_{\mathbb{L}}^{(>1)}$

(ii) $\widetilde{D}^{K_{\mathbb{L}}} \in \widetilde{\mathbb{L}}^{K_{\mathbb{L}}}$ and $\widetilde{D}^{K_{\mathbb{L}}}$ is nonsingular.

(b) The general member $D$ of $\mathbb{L}$ satisfies (a-i) and (a-ii), and goes through $\mathcal{K}_{\mathbb{L}}$ effectively.

\section{RATIONAL LINEAR SYSTEMS ON RATIONAL SURFACES}

In this section, $S$ is a rational nonsingular projective surface.

2.1. Definition. A linear system $\mathbb{L}$ on $S$ is rational if $\operatorname{dim} \mathbb{L} \geq 1$ and the general member of $\mathbb{L}$ is a rational curve.

Given a curve $C \subset S$, it is interesting to ask whether there exists a rational linear system $\mathbb{L}$ on $S$ satisfying $C \in \mathbb{L}$. In this section we show that the existence of $\mathbb{L}$ is equivalent to $C$ being rational and of nonnegative type (cf. 2.5). When $C$ satisfies these conditions, we describe all rational linear systems containing $C$.

We begin by recalling some known facts (2.3 and 2.4). 
2.2. Definition. A pencil $\Lambda$ on $S$ is called a $\mathbb{P}^{1}-$ ruling if it is base-point-free and if its general member is isomorphic to a projective line.

The following fact is a consequence of a well-known result of Gizatullin (see for instance [5, Chap. 2, 2.2] or [3, Sec. 2]). Note that Gizatullin's result is stronger than 2.3. as we are only stating the part of the result which we need.

2.3. Lemma (Gizatullin). Let $\Lambda$ be a $\mathbb{P}^{1}$-ruling on $S$ and let $D \in \Lambda$.

(a) Each irreducible component of $D$ is a nonsingular rational curve.

(b) If $\operatorname{supp}(D)$ is irreducible then $D$ is reduced.

(c) If $\operatorname{supp}(D)$ is reducible then there exists a $(-1)$-component $\Gamma$ of $\operatorname{supp}(D)$ which meets at most two other components of $\operatorname{supp}(D)$; moreover, if $\Gamma$ has multiplicity 1 in the divisor $D$ then there exists another $(-1)$-component of $\operatorname{supp}(D)$ which meets at most two other components of $\operatorname{supp}(D)$.

2.4. Lemma. Consider $C \subset S$ such that $C \cong \mathbb{P}^{1}$ and $C^{2} \geq 0$.

(a) $\operatorname{dim}|C|=C^{2}+1$ and $|C|$ is base-point-free.

(b) For any linear system $\mathbb{L}$ on $S$ such that $C \in \mathbb{L}$ and $\operatorname{dim} \mathbb{L} \geq 1$, the general member of $\mathbb{L}$ is a nonsingular rational curve.

(c) If $C^{2}=0$ then $|C|$ is a $\mathbb{P}^{1}$-ruling.

Proof. Assertions (a) and (c) are well known. Let $\mathbb{L}$ be a linear system on $S$ such that $C \in \mathbb{L}$ and $\operatorname{dim} \mathbb{L} \geq 1$, and consider a general member $D$ of $\mathbb{L}$. Then $D$ is irreducible and reduced (because $\mathbb{L}$ has an element which is irreducible and reduced) and $p_{a}(D)=p_{a}(C)=0$ (because $D$ is linearly equivalent to $C$ ); so $D$ is a nonsingular rational curve.

Let us now turn our attention to the subject matter of this section, i.e., the problem of describing all rational linear systems containing a given curve.

2.5. Definition. Let $C \subset S$ be a curve. Consider the minimal resolution of singularities $\pi=\pi_{K^{C}}: S_{K^{C}} \rightarrow S$ of $C$ (cf. 1.4), and the strict transform $\widetilde{C}=\widetilde{C}^{K^{C}} \subset S_{K^{C}}$ of $C$. Let $\tilde{\nu}(C)$ denote the self-intersection number of $\widetilde{C}$ in $S_{K^{C}}$. If $\tilde{\nu}(C) \geq 0$, we say that $C$ is of nonnegative type. We also define the set

$$
\mathbb{L}_{C}=\left\{\pi_{*}(D)|D \in| \widetilde{C} \mid\right\}
$$

where $\pi_{*}: \operatorname{Div}\left(S_{K^{C}}\right) \rightarrow \operatorname{Div}(S)$ is the homomorphism induced by $\pi=\pi_{K^{C}}$. It is clear that $\mathbb{L}_{C}$ is a linear system on $S$, that $\operatorname{dim} \mathbb{L}_{C}=\operatorname{dim}|\widetilde{C}|$, and that $C \in \mathbb{L}_{C}$.

2.6. Lemma. Let $C \subset S$ be a rational curve.

(a) $\operatorname{dim} \mathbb{L}_{C} \geq 1 \Leftrightarrow \tilde{\nu}(C) \geq 0$

(b) If $\tilde{\nu}(C) \geq 0$, then $\operatorname{dim} \mathbb{L}_{C}=\tilde{\nu}(C)+1$.

(c) If $\tilde{\nu}(C) \geq 0$, then every linear system $\mathbb{L}$ on $S$ satisfying $C \in \mathbb{L} \subseteq \mathbb{L}_{C}$ and $\operatorname{dim} \mathbb{L} \geq 1$ is a rational linear system. 
Proof. Let the notation $\left(\pi=\pi_{K^{C}}: S_{K^{C}} \rightarrow S, \pi_{*}: \operatorname{Div}\left(S_{K^{C}}\right) \rightarrow \operatorname{Div}(S)\right.$ and $\widetilde{C}=$ $\left.\widetilde{C}^{K^{C}} \subset S_{K^{C}}\right)$ be as in 2.5. We have $\operatorname{dim} \mathbb{L}_{C}=\operatorname{dim}|\widetilde{C}|$ and $\tilde{\nu}(C)=\widetilde{C}^{2}$, so assertion (b) follows by applying 2.4(a) to the nonsingular curve $\widetilde{C}$. Part " $\Leftarrow$ " of (a) follows immediately, and the converse is the observation that $\operatorname{dim}|\widetilde{C}| \geq 1$ implies $\widetilde{C}^{2} \geq 0$.

To prove (c), suppose that $\tilde{\nu}(C) \geq 0$ and consider a linear system $\mathbb{L}$ on $S$ satisfying $C \in \mathbb{L} \subseteq \mathbb{L}_{C}$ and $\operatorname{dim} \mathbb{L} \geq 1$. Then there exists a linear system $\mathbb{L}^{\prime}$ on $S_{K^{C}}$ satisfying $\widetilde{C} \in \mathbb{L}^{\prime}$ and $\pi_{*}\left(\mathbb{L}^{\prime}\right)=\mathbb{L}$. Since $\widetilde{C}^{2}=\tilde{\nu}(C) \geq 0$, 2.4(b) implies that the general member of $\mathbb{L}^{\prime}$ is a rational curve; so the general member of $\pi_{*}\left(\mathbb{L}^{\prime}\right)=\mathbb{L}$ is a rational curve.

2.7. Proposition. Let $C \subset S$ be a curve and suppose that $\mathbb{L}$ is a rational linear system on $S$ (cf. 2.11) satisfying $C \in \mathbb{L}$. Then the following hold.

(a) $C$ is a rational curve of nonnegative type.

(b) $\widetilde{C}^{K_{\mathbb{L}}} \in \widetilde{\mathbb{L}}^{K_{\mathrm{L}}}$ and $\widetilde{C}^{K_{\mathbb{L}}}$ is nonsingular.

(c) $C$ goes through $\mathcal{K}_{\mathbb{L}}$ effectively.

(d) The general member $D$ of $\mathbb{L}$ satisfies $\mathcal{K}^{C}=\mathcal{K}^{D}$.

(e) $\mathbb{L} \subseteq \mathbb{L}_{C}$

(f) $K^{C} \subseteq K_{\mathbb{L}}$ and $\tilde{\nu}(C)=\left(\widetilde{C}^{K_{\mathbb{L}}}\right)^{2}+\left|K_{\mathbb{L}} \backslash K^{C}\right| \geq\left|K_{\mathbb{L}} \backslash K^{C}\right|$.

Proof. There is a nonempty Zariski-open subset $U$ of $\mathbb{L}$ such that every element of $U$ is an irreducible rational curve. Pick a pencil $\Lambda \subseteq \mathbb{L}$ such that $C \in \Lambda$ and $\Lambda \cap U \neq \varnothing$; then $\Lambda$ is a rational pencil. Let $\pi_{K_{\Lambda}}: S_{K_{\Lambda}} \rightarrow S$ be the minimal resolution of the base points of $\Lambda$. Then $\widetilde{\Lambda}^{K_{\Lambda}}$ is a $\mathbb{P}^{1}$-ruling and $\widetilde{C}^{K_{\Lambda}}$ is included in the support of an element of $\widetilde{\Lambda}^{K_{\Lambda}}$, so Gizatullin's Theorem 2.3 implies that $\widetilde{C}^{K_{\Lambda}}$ is rational (so $C$ is rational) and nonsingular (so $K^{C} \subseteq K_{\Lambda}$ by (2) ). Let $F \in \widetilde{\Lambda}^{K_{\Lambda}}$ be the element such that $\widetilde{C}^{K_{\Lambda}} \subseteq \operatorname{supp}(F)$. The fact that $1 C \in \Lambda$ implies that

$$
F=1 \widetilde{C}^{K_{\Lambda}}+\sum_{P \in I} a_{P} \widetilde{E}_{P}^{K_{\Lambda}}
$$

for some subset $I \subseteq K_{\Lambda}$ and where $a_{P} \geq 1$ for all $P \in I$.

We claim that $I=\varnothing$. Indeed, suppose the contrary. Then $\operatorname{supp}(F)$ is reducible, so Gizatullin's Theorem implies that $\operatorname{supp}(F)$ has a $(-1)$-component $\Gamma$, and that if $\Gamma$ has multiplicity 1 in $F$ then $\Gamma$ is not the only $(-1)$-component of $\operatorname{supp}(F)$. This together with (5) imply that there exists $P \in I$ such that $\left(\widetilde{E}_{P}^{K_{\Lambda}}\right)^{2}=-1$; as $P \in K_{\Lambda}$ and $\widetilde{E}_{P}^{K_{\Lambda}}$ is vertical, this contradicts (4), and proves that $I=\varnothing$. So:

$$
\widetilde{C}^{K_{\Lambda}} \in \widetilde{\Lambda}^{K_{\Lambda}} \text {. }
$$

It follows that $\left(\widetilde{C}^{K_{\Lambda}}\right)^{2}=0$ in $S_{K_{\Lambda}}$, because $\widetilde{\Lambda}^{K_{\Lambda}}$ is a base-point-free pencil. As $\widetilde{C}^{K_{\Lambda}}$ is also nonsingular, $C$ is of nonnegative type and (a) is proved.

Since $\widetilde{C}^{K_{\Lambda}} \in \widetilde{\Lambda}^{K_{\Lambda}}$ and $\widetilde{C}^{K_{\Lambda}}$ is nonsingular, 1.11 implies that $\mathcal{K}^{C}=\mathcal{K}_{\Lambda}^{(>1)}$. As the general member $D$ of $\Lambda$ satisfies $\mathcal{K}^{D}=\mathcal{K}_{\Lambda}^{(>1)}$ by 1.11, we get $\mathcal{K}^{D}=\mathcal{K}^{C}$. So we have shown that, for any pencil $\Lambda$ satisfying $C \in \Lambda \subseteq \mathbb{L}$ and $\Lambda \cap U \neq \varnothing$, the general member 
$D$ of $\Lambda$ satisfies $\mathcal{K}^{D}=\mathcal{K}^{C}$. Consequently, $\left\{D \in \mathbb{L} \mid \mathcal{K}^{D}=\mathcal{K}^{C}\right\}$ is a dense subset of $\mathbb{L}$; together with the fact (1.11) that $\mathcal{K}^{D}=\mathcal{K}_{\mathbb{L}}^{(>1)}$ for general $D \in \mathbb{L}$, this implies

$$
\mathcal{K}^{C}=\mathcal{K}_{\mathbb{L}}^{(>1)} .
$$

Then assertions (b) and (d) follow from (7) and 1.11, and assertion (c) follows from $\widetilde{C}^{K_{\mathbb{L}}} \in \widetilde{\mathbb{L}}^{K_{\mathbb{L}}}$ and 1.9. By (77) we have $e_{P}(C)=e_{P}(\mathbb{L})$ for all $P \in K^{C}$; this together with 1.9 implies that $\widetilde{C}^{K^{C}} \in \widetilde{\mathbb{L}}^{K^{C}}$, hence $\widetilde{\mathbb{L}}^{K^{C}} \subseteq\left|\widetilde{C}^{K^{C}}\right|$. It follows that $\pi_{*}\left(\widetilde{\mathbb{L}^{K}}\right) \subseteq$ $\pi_{*}\left(\left|\widetilde{C}^{K^{C}}\right|\right)$, where $\pi_{*}: \operatorname{Div}\left(S_{K^{C}}\right) \rightarrow \operatorname{Div}(S)$ is the homomorphism induced by $\pi=$ $\pi_{K^{C}}: S_{K^{C}} \rightarrow S$. As $\pi_{*}\left(\widetilde{\mathbb{L}}^{K^{C}}\right)=\mathbb{L}$ and (by definition) $\pi_{*}\left(\left|\widetilde{C}^{K^{C}}\right|\right)=\mathbb{L}_{C}$, (e) is true.

We have $K^{C} \subseteq K_{\mathbb{L}}$ by (17), and (c) implies that $e_{P}(C)=1$ for all $P \in K_{\mathbb{L}} \backslash K^{C}$. Consequently, $\tilde{\nu}(C)=\left(\widetilde{C}^{K^{C}}\right)^{2}=\left(\widetilde{C}^{K_{\mathbb{L}}}\right)^{2}+\left|K_{\mathbb{L}} \backslash K^{C}\right|$. Pick any $D \in \widetilde{\mathbb{L}}^{K_{\mathbb{L}}} \backslash\left\{\widetilde{C}^{K_{\mathbb{L}}}\right\}$, then $\left(\widetilde{C}^{K_{\mathbb{L}}}\right)^{2}=\widetilde{C}^{K_{\mathbb{L}}} \cdot D \geq 0$ and (f) is proved.

2.8. Theorem. For a curve $C \subset S$, the following are equivalent:

(a) $\mathbb{L}_{C}$ is a rational linear system;

(b) there exists a rational linear system $\mathbb{L}$ on $S$ such that $C \in \mathbb{L}$;

(c) $C$ is rational and $\operatorname{dim} \mathbb{L}_{C} \geq 1$;

(d) $C$ is rational and $\tilde{\nu}(C) \geq 0$.

Moreover, if conditions $(\mathrm{a}-\mathrm{d})$ are satisfied then the following hold:

(e) For a linear system $\mathbb{L}$ on $S$ satisfying $C \in \mathbb{L}$ and $\operatorname{dim} \mathbb{L} \geq 1$,

$\mathbb{L}$ is rational $\Longleftrightarrow \mathbb{L} \subseteq \mathbb{L}_{C}$.

(f) $\operatorname{dim} \mathbb{L}_{C}=\tilde{\nu}(C)+1, \mathcal{K}_{\mathbb{L}_{C}}=\mathcal{K}^{C}$ and $\widetilde{\mathbb{L}}_{C}^{K^{C}}=\left|\widetilde{C}^{K^{C}}\right|$.

Proof. Suppose that $\mathbb{L}$ is a rational linear system on $S$ such that $C \in \mathbb{L}$. By 2.7, we obtain that $C$ is rational and that $\mathbb{L} \subseteq \mathbb{L}_{C}$ (so $\operatorname{dim} \mathbb{L}_{C} \geq 1$ ). So (b) implies (c), and this also proves implication " $\Rightarrow$ " in statement $(\mathrm{e})$.

Equivalence $(\mathrm{c}) \Leftrightarrow(\mathrm{d})$ is $2.6(\mathrm{a})$, implication (c and $\mathrm{d}) \Rightarrow(\mathrm{a})$ is the case $\mathbb{L}=\mathbb{L}_{C}$ of $2.6(\mathrm{c})$, and $(\mathrm{a}) \Rightarrow(\mathrm{b})$ is obvious. So (a-d) are equivalent.

Now assume that $(\mathrm{a}-\mathrm{d})$ are satisfied. Implication " $\Leftarrow$ " in statement (e) is a consequence of 2.6(c), so there only remains to prove (f). Equality $\operatorname{dim} \mathbb{L}_{C}=\tilde{\nu}(C)+1$ is 2.6(b). Observe that there can be at most one linear system $\mathbb{G}$ on $S_{K^{C}}$ satisfying

the general member of $\mathbb{G}$ is irreducible and $\pi_{*}(\mathbb{G})=\mathbb{L}_{C}$,

where $\pi=\pi_{K^{C}}: S_{K^{C}} \rightarrow S$; as $\widetilde{\mathbb{L}}_{C}^{K^{C}}$ and $\left|\widetilde{C}^{K^{C}}\right|$ are two such linear systems, we get $\widetilde{\mathbb{L}}_{C}^{K^{C}}=\left|\widetilde{C}^{K^{C}}\right|$. This implies that $\widetilde{\mathbb{L}}_{C}^{K^{C}}$ is base-point-free (because $\left|\widetilde{C}^{K^{C}}\right|$ is base-pointfree by 2.4), so all base points of $\mathbb{L}_{C}$ are in $K^{C}$, i.e., $K_{\mathbb{L}_{C}} \subseteq K^{C}$. On the other hand, 2.7(b) together with 1.11 gives $\mathcal{K}^{C}=\mathcal{K}_{\mathbb{L}_{C}}^{(>1)}$ (which was also noted in (17)); this and $K_{\mathbb{L}_{C}} \subseteq K^{C}$ imply $\mathcal{K}_{\mathbb{L}_{C}}=\mathcal{K}^{C}$, which completes the proof. 


\section{REFERENCES}

[1] Alberich-Carramiñana, M., Geometry of the plane Cremona maps, Springer Lecture Notes in Math. 1769 (2002).

[2] Daigle, D. and Melle-Hernández, A., Linear systems associated to unicuspidal rational plane curves, in preparation.

[3] Gizatullin, M. H., Affine surfaces that can be augmented by a nonsingular rational curve, Izv. Akad. Nauk SSSR Ser. Mat. 34 (1970), 778-802.

[4] Manin, Y. I., Cubic forms: algebra, geometry, arithmetic (Translated from Russian by M. Hazewinkel), North-Holland Mathematical Library, Vol. 4 (1974).

[5] Miyanishi, M., Curves on rational and unirational surfaces, Tata Inst. Fund. Res. Lectures on Math. and Phys. 60 (1978).

Department of Mathematics and Statistics, University of Ottawa, Ottawa, Canada K1N $6 \mathrm{~N} 5$

iCMAT (CSIC-UAM-UC3M-UCM) Dept. of Algebra, Facultad de Ciencias Matemáticas, Universidad Complutense, 28040, Madrid, Spain

E-mail address: ddaigle@uottawa.ca

E-mail address: amelle@mat.ucm.es 